\title{
I want to break free: liberation from noninvasive ventilation
}

\author{
Stefano Nava ${ }^{1}$ and Begum Ergan ${ }^{2}$
}

Affiliations: ${ }^{1}$ Respiratory and Critical Care Unit, Dept of Clinical, Integrated and Experimental Medicine (DIMES), Sant'Orsola-Malpighi Hospital, Alma Mater University, Bologna, Italy. ${ }^{2}$ Dept of Pulmonary and Critical Care, Faculty of Medicine, Dokuz Eylul University, Izmir, Turkey.

Correspondence: S. Nava, Sant'Orsola-Malpighi Hospital, via Massarenti n.9 Bologna, Bologna 40138, Italy. E-mail: stefanavadgmail.com

@ERSpublications

Weaning from noninvasive ventilation amongst patients with acute hypercapnic respiratory failure http://ow.ly/o3eq30clY6o

Cite this article as: Nava S, Ergan B. I want to break free: liberation from noninvasive ventilation. Eur Respir J 2017; 50: 1700674 [https://doi.org/10.1183/13993003.00674-2017].

\begin{abstract}
A lot of emphasis has been placed on the weaning process from invasive mechanical ventilation, since prolonged ventilation has been associated with increased length of intensive care unit stay, increased levels of complications and increased costs. Weaning in this respect may be considered as an "all or none" phenomenon, since the patients are abruptly removed from any form of ventilator support after extubation unless they are placed on noninvasive ventilation (NIV). In patients with acute hypercapnic respiratory failure (AHRF), particularly those with chronic obstructive pulmonary disease (COPD), the use of NIV is considered to be the first line treatment. However, no systematic studies have so far been performed in this population, except for some small observational investigations. The study by SELLARES et al. [1] in this issue of the European Respiratory Journal is therefore a welcome addition to the field in order to finally shed some light on the subject.
\end{abstract}

In their trial Sellares et al. [1] randomised 120 COPD patients (with no previous history of domiciliary ventilation) who were admitted for AHRF and treated with NIV. When this was solved and patients could tolerate spontaneous breathing (i.e. $\mathrm{pH}>7.35$ ) for $4 \mathrm{~h}$, they were allocated either to receive nocturnal NIV for three more nights or for discontinuation of NIV. Except for a shorter median stay in the intermediate respiratory care unit for the discontinuation group, no differences were observed in the main outcome (relapse of AHRF). Being an "intermittent" technique of ventilator support, where relatively long intervals of spontaneous breathing are allowed, NIV may give us the unique opportunity to better understand the level of autonomy of patients during the weaning phase and, even indirectly, to shed light on the physiology of the AHRF recovery period.

Nocturnal hypoventilation has been claimed to be one of the factors interfering with the weaning process. In fact, in Sellares's study, those patients randomised to be treated with NIV for three consecutive nights after they reached clinical stability did not have a reduced occurrence of relapse versus those patients abruptly liberated from mechanical ventilation. Furthermore, nocturnal hypoventilation (i.e. arterial carbon dioxide tension $\left(\mathrm{PaCO}_{2}\right)$ increase during sleep $>10 \mathrm{mmHg}$ ) is present in up to $40 \%$ of COPD patients, who can live for years without any form of respiratory support despite a night time increase in $\mathrm{PaCO}_{2}$ during sleep. Therefore, it is likely that while hypoventilation worsens during an episode of AHRF, once these patients regain compensation in $\mathrm{pH}$ they return very quickly to their original condition. One limitation of this study is that no assessment of the presence of nocturnal hypoventilation was performed, such that the

Received: March 312017 | Accepted after revision: April 142017

Conflict of interest: None declared.

"I want to break free" from the Queen album “The Works", EMI/Parlophone, 1984.

Copyright @ERS 2017 
two groups of patients may not have been balanced when randomisation was performed. Indeed, it has been shown that the NIV settings, rather than the mode, may also determine the outcome for the patient but no data about this was provided in the paper. Patients requiring NIV may have an imbalance between the load on the respiratory system and its capacity that theoretically may interfere with the weaning process. The abrupt removal of NIV resulted in a new relapse in $13 \%$ of the patients randomised into this group, suggesting that most patients recovering from AHRF may reach an equilibrium between load and capacity much faster that has been suggested in a recent physiological study [2].

Sellares's study basically demonstrates that weaning from NIV is probably not limited either by nocturnal hypoventilation or persistent high work of breathing when equilibrium in gas exchange has been reached. As such, the logical consequence is that once the patient is able to maintain a $\mathrm{pH}>7.35$ during a spontaneous breathing trial of $4 \mathrm{~h}$, then NIV should be suspended. This limit was arbitrarily chosen by the authors as a criterion of enrollment and obviously seems to be a valid threshold in light of their results. However, it is worthwhile to note that the baseline level of $\mathrm{pH}$ when NIV was started was moderately severe (median $\mathrm{pH}$ 7.29) and therefore it needs to eventually be validated in more severe episodes of AHRF. In addition, another important point to consider while interpreting the results of the present study is its generalizability. While monocentric, the study was performed in an experienced centre for NIV and, for that reason, both the decision on weaning and the weaning procedure itself were probably carried out to the highest standards. This is also confirmed by the findings of the relatively short period of NIV use after randomisation and the high rate of successful weaning on first attempt. Therefore, caution must be taken while evaluating the results as they may not be valid for other centres.

Overall this elegant study poses another important question: should weaning from NIV be performed even before normal values of $\mathrm{pH}$ are reached? Girault et al. [3] have, for example, shown that a successful extubation to spontaneous breathing can be performed, at least in a subset of COPD patients, even when $\mathrm{pH}$ at the end of a T-piece trial is $<7.35$. Alternatively, different strategies of liberation from NIV can be imagined, such as a progressive daily decrease in the level of support (i.e. $2 \mathrm{cmH}_{2} 0$ every $4 \mathrm{~h}$ during day time) or an increase in the length of spontaneous breathing between NIV intervals, albeit as a faster rate than that used by DAMAS et al. [4], where progressive reduction of NIV duration was accomplished over 3 consecutive days. These two options might eventually be started much earlier than in the Sellares study (i.e. after the first $24 \mathrm{~h}$ of ventilation), as long as a satisfactory level of gas exchange is reached during NIV and spontaneous breathing autonomy is feasible without dyspnoea or excessive discomfort.

We clinicians usually want to be "on the safe-side" during AHRF and, therefore, significant numbers of patients might be receiving NIV for longer durations than needed. Similar to the present study findings, LuN et al. [5] compared immediate discontinuation with stepwise decrease in NIV duration over 3 days and found no difference between the two groups in respect to treatment failure. Abrupt discontinuation can therefore be a reasonable option to try in clinically stable, spontaneously breathing COPD patients. In addition, the advantage of easy reinstatement of NIV gives the clinician an ideal opportunity to proceed. However, it should also be kept in mind that the most important factor for NIV success is the patient's clinical status and disease severity. Whether different protocols for discontinuing NIV for patient groups with different levels of AHRF severity would be an option in the near future is another subject to investigate.

Another important statistical point to consider is that the authors have used a type-II error of 0.20 for sample size calculation. A beta error occurs when the null hypothesis is incorrectly accepted, assuming no difference between treatment arms although they actually differ. Accepting a 0.20 beta error rate means the study was powered to have an $80 \%$ likelihood of detecting a difference between treatment arms and if the objective of a study is to demonstrate that treatments are equivalent, the type-II error rate should probably be set lower [6]. Despite some limitations, the Sellares study gives us an important clinical message; however, it might be considered as only the first step towards a better understand of the complex problem of regaining spontaneous autonomy after an episode of AHRF.

\section{References}

1 Sellares J, Ferrer M, Antoni A, et al. Discontinuing noninvasive ventilation in severe chronic obstructive pulmonary disease exacerbations: a randomised controlled trial. Eur Respir J 2017; 50: 1601448.

2 Ceriana P, Vitacca M, Carlucci A, et al. Changes of respiratory mechanics in COPD patients from stable state to acute exacerbations with respiratory failure. COPD 2017; 14: 150-155.

3 Girault C, Bubenheim M, Abroug F, et al. Noninvasive ventilation and weaning in patients with chronic hypercapnic respiratory failure. Am J Respir Crit Care Med 2011; 184: 672-679.

4 Damas C, Andrade C, Araújo AP, et al. Desmame de ventilação não invasiva: experiência com períodos de descontinuação [Weaning from non-invasive positive pressure ventilation: experience with progressive periods of withdrawal]. Rev Port Pneumol 2008; 14: 49-53.

5 Lun CT, Chan VL, Leung WS, et al. A pilot randomized study comparing two methods of non-invasive ventilation withdrawal after acute respiratory failure in chronic obstructive pulmonary disease. Respirology 2013; 18: 814-819.

6 Scales DC, Rubenfeld GD. Estimating sample size in critical care clinical trials. J Crit Care 2005; 20: 6-11. 